\title{
Neuronutrição na depressão e transtorno de ansiedade
}

\author{
Neuronutrition in depression and anxiety disorder \\ Neuronutrición en trastorno de depresión y ansiedad
}

\author{
Gabriela Silva Baklizi \\ ORCID: https://orcid.org/0000-0002-5252-2172 \\ Centro Universitário de Brasília, Brasil \\ E-mail: gabriela.baklizi@sempreceub.com \\ Beatriz Carvalho Bruce \\ ORCID: https://orcid.org/0000-0002-9299-9055 \\ Centro Universitário de Brasília, Brasil \\ E-mail: beatriz.carvalho@sempreceub.com \\ Ana Cristina de Castro Pereira Santos \\ ORCID: https://orcid.org/0000-0001-8622-7879 \\ Centro Universitário de Brasília, Brasil \\ E-mail: ana.cristinasantos@ ceub.edu.br
}

\begin{abstract}
Resumo
A ansiedade e depressão são doenças cada vez mais presentes nos dias de hoje, consideradas as doenças do século, afetam drasticamente a qualidade de vida da população. Através da neuronutrição é possível analisar hábitos alimentares e sua relação com o nosso desempenho cognitivo, analisando desde a fisiologia, bioquímica e microbiota até a influência da nutrição diretamente ao desenvolvimento desses transtornos mentais. Este estudo teve como objetivo compreender, por meio de uma revisão da literatura, o papel da neuronutrição na depressão e transtorno de ansiedade. Trata-se de uma revisão sistemática, com a consulta sobre o tema a partir de revistas e periódicos científicos e à base de dados como: PubMed, SCIELO, Google Acadêmico e CAPES, a partir dos seguintes descritores: alimentação, ansiedade, depressão, nutrientes, ômega 3, vitamina D, cérebro e intestino, vitaminas e sistema nervoso. Através do estudo foi possível analisar como a nutrição influencia tanto na prevenção quanto no controle e amenização dos sintomas.
\end{abstract}

Palavras-chave: Ansiedade; Depressão; Nutrientes.

\begin{abstract}
Anxiety and depression are diseases increasingly present today, considered the diseases of the century, drastically affect the quality of life of the population. Through neuronutrition it is possible to analyze eating habits and their relationship with our cognitive performance, analyzing from physiology, biochemistry and microbiota to the influence of nutrition directly on the development of these mental disorders. This study aimed to understand, through a literature review, the role of neuronutrition in depression and anxiety disorder. from data such as: PubMed, SCIELO, Academic Google and CAPES, from the following descriptors: diet, anxiety, depression, nutrients, omega 3, vitamin D, brain and intestine, vitamins and nervous system. Through the study, it was possible to analyze how nutrition influences both the prevention and control and alleviation of symptoms.
\end{abstract}

Keywords: Anxiety; Depression; Nutrients.

\section{Resumen}

La ansiedad y la depresión son enfermedades cada vez más presentes en la actualidad, consideradas las enfermedades del siglo, afectan drásticamente la calidad de vida de la población. A través de la neuronutrición es posible analizar los hábitos alimentarios y su relación con nuestro desempeño cognitivo, analizando desde la fisiología, bioquímica y microbiota hasta la influencia de la nutrición directamente en el desarrollo de estos trastornos mentales. Este estudio tuvo como objetivo comprender, a través de una revisión de la literatura, el papel de la neuronutrición en la depresión y el trastorno de ansiedad. A partir de datos como: PubMed, SCIELO, Academic Google y CAPES, a partir de los siguientes descriptores: dieta, ansiedad, depresión, nutrientes, omega 3 , vitamina D, cerebro e intestino, vitaminas y sistema nervioso. A través del estudio, fue posible analizar cómo la nutrición influye tanto en la prevención como en el control y alivio de los síntomas.

Palabras clave: Ansiedad; Depresión; Nutrientes. 


\section{Introdução}

Neuronutrição é uma área que está sendo estudada recentemente, relacionando com a influência de uma alimentação saudável com a saúde mental em todos os ciclos da vida. O consumo de nutrientes adequados tem o objetivo de ajudar no equilíbrio da saúde, fazendo com que evite doenças de transtornos mentais. A neuronutrição destaca como os alimentos influenciam o cérebro e suas funções rotineiras (Fernandes, et al., 2021).

Nos dias atuais, estão vivendo uma geração de pessoas ansiosas, depressivas e estressadas, mas o que poucos sabem é que ter uma alimentação equilibrada, com um planejamento dietético adequado, pode ajudar no controle da saúde mental. A ansiedade é um sentimento vazio que está interligado ao medo e a preocupação excessiva de algo que ainda vai acontecer. Durante as crises de ansiedade é comum que o indivíduo tenha sintomas físicos, como sudorese e arritmia cardíaca (D’ávila, et al., 2020).

Em relação a depressão, é uma doença mental crônica que está presente na atual geração, um problema difícil de lidar com características que oscilam a um estado de ânimo irritável, a uma falta de motivação e diminuição de atividades diárias simples de serem realizadas. É marcada por sintomas que alteram o apetite, o sono, tensão muscular, cansaço, na maioria das vezes na parte da manhã, baixa autoestima, sentimento de culpa, dificuldades para pensar ou se concentrar, indecisão, ideias de morte e/ou de suicídio e tentativas de suicídio (Rufino, et al., 2018).

De acordo com a Organização Mundial da Saúde (OMS), 18,6 milhões de brasileiros sofrem com algum distúrbio relacionado à ansiedade. Com o elevado número de casos de transtorno de ansiedade no Brasil, mudança dos hábitos e estilo de vida da população estão interligados com uma má alimentação, tendo em vista que uma boa alimentação contribui para uma produção dos neurotransmissores responsáveis por promover o bem-estar emocional (Yogi, et al., 2019).

A alimentação equilibrada previne e trata a ansiedade e a depressão, minimizando os sintomas e a individualidade negativa das doenças, ou seja, a prática de uma alimentação funcional, além de não invasiva, se mostra uma excelente opção para melhor prognóstico da doença (Gonçalves, et al., 2016). Alguns nutrientes com o potencial ansiolítico do L-triptofano, ômega 3, magnésio e das vitaminas do complexo B, apresentaram alta eficácia no combate a estes transtornos psicológicos (Andrade, et al., 2018).

Diante do exposto, o objetivo deste estudo foi analisar a relação da depressão e transtorno da ansiedade com a neuronutrição.

\section{Metodologia}

Trata-se de um estudo de revisão bibliográfica narrativa com a consulta sobre o tema a partir de revistas e periódicos científicos e à base de dados como: PubMed, SCIELO, Google Acadêmico e CAPES. Foram selecionados artigos científicos de publicação em periódicos nacionais e internacionais, nos idiomas português e inglês, dos últimos 12 anos. Para a pesquisa foram utilizados os descritores (DeCS): “alimentação", “ansiedade”, “depressão”, “nutrientes” “ômega 3”, "vitamina D”, "cérebro e intestino", "vitaminas e sistema nervoso", "food", "anxiety", "depression”, "nutrition", "vitamin D”, "brain and intestine”, vitamins and nervous system".

A leitura e abordagem do tema escolhido foi feita a partir de títulos relacionados à ansiedade, depressão e nutrição, entre outros descritores. Em seguida, empreendeu-se uma leitura minuciosa e crítica dos manuscritos para identificação dos núcleos de sentido de cada texto, e posterior agrupamento de subtemas que sintetizassem as produções. Para a seleção dos artigos foram incluídas as bibliografias que relacionam neuronutrição a transtorno de ansiedade e depressão e descartadas aquelas que não atenderam a temática.

Fluxograma mediante os critérios de inclusão e exclusão de artigos, foram selecionados 33 artigos para a presente revisão. 
Figura 1. Resumo da busca de dados para a presente revisão. Brasília-DF, 2021.

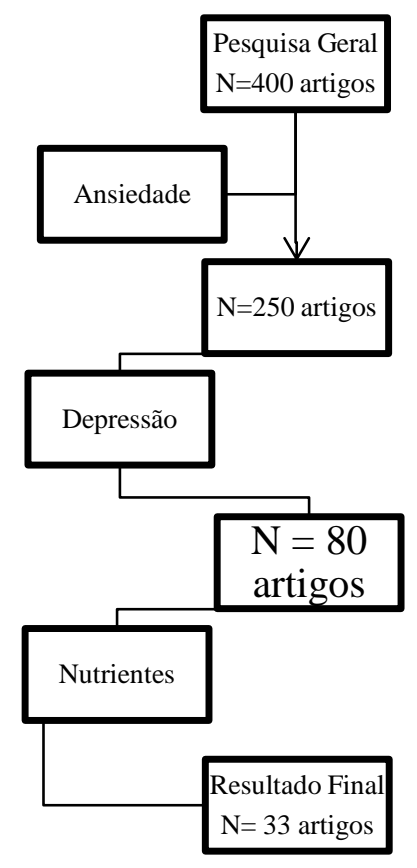

Fonte: Autores.

\section{Resultados e Discussão}

\subsection{Ansiedade e Depressão}

Segundo a Organização Mundial da Saúde (OMS) (2017), cerca de 3,6\% da população mundial possui algum tipo de transtorno de ansiedade, sendo mais comum em pessoas do sexo feminino (4,6\%), do que em pessoas do sexo masculino (2,6\%). Este estudo mostrou que o Brasil é o país com mais cidadãos com transtorno de ansiedade, cerca de 9,3\% dos envolvidos.

Através de um extenso período de tempo a ansiedade pode ser sentida por meio da ausência de uma ameaça física. O transtorno de ansiedade é definido como um estado emocional em indivíduos que não conseguem controlar seus variados sinais. Mais uma vez a desregulação dos neurotransmissores afetam o indivíduo de maneira negativa, mas não é apenas esse motivo que está relacionado a ansiedade, contribuições biopsicossociais, até mesmo o estresse do dia a dia e condições genéticas também podem desencadear a ansiedade (Hartley \& Phelps, 2012).

A ansiedade associada ao comportamento alimentar faz com que o indivíduo recorra aos alimentos em períodos mais intensos, principalmente em momentos de sentimentos de medo e insegurança. Isso acaba acontecendo sem a intenção de ir até o alimento como forma de recompensa, conforto ou prazer (Aro, et al., 2021).

Estudos científicos comprovam que um método eficaz para o controle do transtorno de ansiedade compõe uma alimentação saudável rica em nutrientes como o triptofano, ômega-3, o magnésio, vitaminas do complexo B, vitamina C, vitamina A, minerais como cálcio, zinco e ferro (Rocha \& Myva, 2020).

Segundo Diniz et al. (2020) a depressão é descrita por tristeza, baixa autoestima, oscilações entre sentimento de culpa e perda ou diminuição de interesse e prazer pela vida, e também distúrbios do sono ou do apetite. Pode ser causada por fatores genéticos, biológicos e externos.

De acordo com o Almada et al. (2014), nos dias de hoje existem vários estudos que comprovam as alterações químicas no cérebro da pessoa que possui depressão, os estudos mostram a ligação com neurotransmissores, substâncias que transmitem impulsos nervosos para as células. 
Um dos principais neurotransmissores formado por moléculas pequenas são o 5-hidroxitriptamina (5-HT) e norepinefrina (NE). A 5-Hidroxitriptamina está associada aos transtornos psiquiátricos, visto que na depressão há redução dos níveis desse neurotransmissor no sistema nervoso central. Já a norepinefrina também há redução dos níveis, e é um dos mais importantes neurotransmissor dos neurônios simpáticos, também produzida via sistema nervoso central ela é classificada como catecolamina endógena (Diniz, et al., 2020).

\subsection{Fisiologia e Bioquímica da ansiedade e depressão}

Os transtornos de humor e ansiedade são caracterizados por uma variedade de distúrbios neuroendócrinos, neurotransmissores e neuroanatômicos. Os centros cognitivos superiores do cérebro residem no lobo frontal. O córtex pré-frontal (PFC) é responsável por funções executivas, como planejamento, tomada de decisão, previsão de consequências para comportamentos potenciais e compreensão e moderação do comportamento social. As estruturas cerebrais de processamento emocional são chamadas de sistema límbico. O córtex límbico integra os componentes sensoriais, afetivos e cognitivos da dor e processa informações sobre o estado corporal interno (Martin, et al., 2009).

Além da atividade de cada região do cérebro, também é importante considerar os neurotransmissores que fornecem a comunicação entre essas regiões. $\mathrm{O}$ aumento da atividade nas regiões cerebrais de processamento de emoções em pacientes com transtorno de ansiedade, pode resultar da diminuição da sinalização inibitória pelo ácido $\gamma$-amino-butírico (GABA) ou aumento da neurotransmissão excitatória pelo glutamato (Calhoon \& Tye, 2015).

Quando há algum fator de estresse, um sinal é enviado em forma de estímulo sensorial. No tálamo a informação é enviada em direção a amígdala e hipocampo. A amígdala é responsável pela expressão de medo e agressão, desempenha um papel na formação e recuperação de memórias emocionais relacionadas ao medo. O complexo amigdalóide funciona como o "centro de análise das circunstâncias" - por meio da comparação de experiências/memórias vividas com a experiência atual, gerando o aumento de alerta. Conforme a figura 2, a ativação de forma crônica da amígdala torna o indivíduo mais reativo ao estresse, portanto o mesmo estímulo estressor é capaz de gerar uma resposta muito mais acentuada, gerando uma alteração na percepção emocional (Martin, et al., 2009).

Figura 2. Mecanismo da amígdala no circuito do medo.

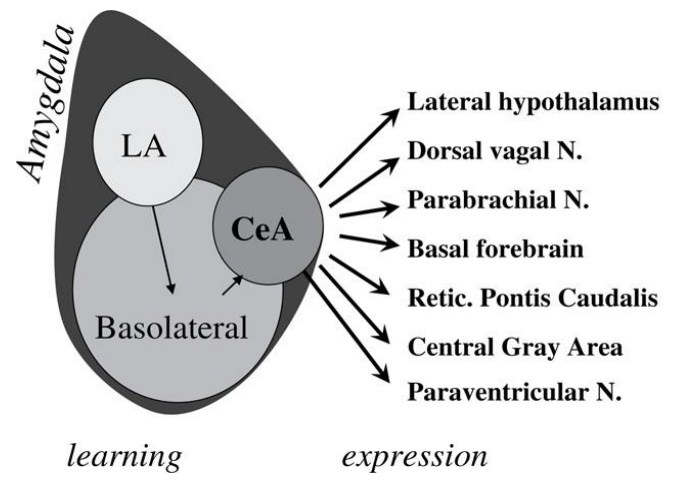

\section{Fear / Panic Symptoms:}

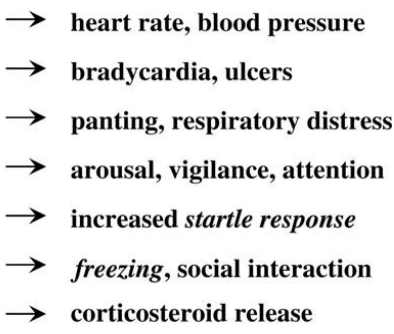

Fonte: Martin, et al. (2009).

O hipocampo fica responsável por sua contextualização, tem controle inibitório tônico sobre o sistema de resposta ao estresse hipotalâmico e desempenha um papel no feedback negativo para o eixo hipotálamo-hipófise-adrenal (HPA). Em uma situação de estresse ocorre a ativação do eixo HPA onde é liberado o hormônio liberador de corticotrofina nos portais hipofisários, 
gerando estímulo à liberação do hormônio adrenocorticotrófico na hipófise anterior. O hormônio adrenocorticotrófico estimula a produção e liberação do cortisol no córtex adrenal na corrente sanguínea (Tafet \& Nemeroff, 2020).

O volume hipocampal e a neurogênese (crescimento de novas células) nessa estrutura têm sido implicados na sensibilidade e resiliência ao estresse em relação aos transtornos de humor e ansiedade (Martin, et al., 2009).

No processo de resposta hormonal pode ocorrer a habituação ou a facilitação. Na habituação o estímulo estressor gera uma resposta menor de cortisol, porém na facilitação o mesmo estímulo estressor gera um aumento do hormônio do estresse, consequentemente, resultando em sintomas mais intensos (Tafet \& Nemeroff, 2020)

\subsection{Eixo intestino cérebro}

Há uma percepção crescente de que o eixo intestino-cérebro e sua regulação pela microbiota pode desempenhar um papel fundamental na base biológica e fisiológica do neurodesenvolvimento, relacionado às doenças neurodegenerativas (Foster, 2013).

O equilíbrio do intestino e da microbiota intestinal exerce influência na nossa parte cerebral e imunológica. Estresse, alimentação, uso de medicamentos, gestação, desenvolvimento na primeira infância, entre outros, são alguns fatores de risco que contribuem para a desordem da sua funcionalidade (Foster \& Mcvey, 2013).

A via de comunicação entre intestino e cérebro é feita pelo o nervo vargus, é bidirecional cujo alterações neurais e a microbiota intestinal estão sempre trocando informações. Quando acontece uma alteração de bactérias no intestino pode ocorrer uma degradação do muco protetor, principalmente quando há uma diminuição no consumo de fibras, aumentando o risco de gerar uma hipermeabilidade intestinal, favorecendo a passagem de endotoxinas para o organismo, gerando um aumento do processo inflamatório (Meroni, et al., 2019; Ekstrand, et al., 2020).

O equilíbrio da microbiota envolve quantidade de bactérias e preservação da diversidade bacteriana em proporções adequadas e ambiente metabólico (ph, temperatura, metabólitos). Assim, fatores como baixo consumo de fibras, estresse, uso de antibióticos, sedentarismo, tabagismo, etilismo, excesso de proteínas, alto consumo calórico, açúcar e gorduras, podem resultar em uma disbiose intestinal, podendo ocorrer um processo inflamatório (Foster \& Mcvey, 2013).

A dieta tem sido associada ao risco de desenvolver depressão e ansiedade; há efeitos diretos dos componentes da dieta que podem mediar essa relação. Além disso, o uso de intervenção dietética pode ser uma alternativa atraente e econômica ou terapia adjuvante para tratar clinicamente esses distúrbios (Bear, et al., 2020).

\subsection{Relação entre nutrição, hábito de vida e distúrbios de humor}

\subsubsection{Influência da nutrição}

A nutrição é um pré-requisito para uma vida saudável desde os primeiros estágios da vida (período de gestação), sabemos que ela está ligada diretamente com a comida, por isso o peso da importância no crescimento e desenvolvimento do ser humano. Portanto, uma boa alimentação é de grande importância na nutrição do indivíduo, com ela podemos alcançar uma saúde cerebral saudável e boa função neurocognitiva (Calhoon, 2015). Um estudo transversal feito em 3.363 participantes adultos iranianos, demonstrou uma redução de 49\% do risco de ansiedade em pessoas que seguem um padrão alimentar saudável (Saneei, et al., 2016).

A dieta mediterrânea apresentou um efeito protetor para ansiedade e depressão. O maior consumo de grãos integrais, vegetais e baixa ingestão calórica apresentaram resultados significativos relacionados à melhora em quadros depressivos. Uma dieta menos saudável foi significativamente associada à gravidade dos sintomas de ansiedade e depressão (Smith, et al., 2019).

Os precursores dos neurotransmissores são ácidos graxos ômega-3, vitamina B e aminoácidos, portanto, sua deficiência está ligada aos transtornos mentais, sendo de extrema importância o consumo de alimentos fontes dessas vitaminas. A deficiência 
dos mesmos pode ser bastante prejudicial para a piora dos sintomas de doenças neurodegenerativas ou transtornos mentais (Fernandes, et al., 2020)

Uma boa alimentação é essencial no combate e controle da ansiedade e depressão, pois estimula a liberação dos neurotransmissores no sistema nervoso central (SNC) e periférico (SNP), sintetizando mais serotonina, noradrenalina e dopamina, que são responsáveis por dar ao cérebro sensação de bem-estar, aumentando o bom humor e sensação de saciedade (Gonçalves, et al., 2017).

\subsection{2 Álcool}

A Organização Mundial da Saúde (OMS) publicou em 2018 um estudo sobre o consumo de álcool no mundo e, com base nos resultados, observou-se um maior percentual de mortes entre os homens do que entre as mulheres, por causas relacionadas ao álcool.

Neste momento da pandemia da Covid-19 foi realizada uma pesquisa pela Organização Pan-Americana da Saúde (Opas) (2020) em que afirma uma preocupação levantada como o aumento do consumo de álcool durante o isolamento social. Assim, temos o álcool, que é substância psicotrópica e atua no sistema nervoso central, e seu consumo está associado a vários transtornos mentais. E durante o isolamento social foi propício o aumento dessa substância que pode desencadear episódios de depressão e ansiedade, além de um menor bem-estar mental (Garcia \& Sanchez, 2020).

O uso exacerbado do álcool traz grandes prejuízos e muitas consequências para o organismo da pessoa que o consome, uma delas é a alteração na absorção de nutrientes, como as vitaminas (complexo B, principalmente a tiamina e o folato, A, D e K) e minerais (magnésio, zinco e cálcio), e o estoque desses nutrientes acabam sendo diminuídos (Roth, et al., 2019).

\subsubsection{Sono}

Cada ser humano possui um tempo certo de descanso de acordo com a sua idade, se não estiver fazendo esse descanso de maneira correta isso pode afetar o corpo fisicamente, e também psicologicamente. Dormir pouco traz grandes problemas, da mesma forma que o excesso de horas dormidas afeta negativamente (Cardoso \& Chagas, 2019).

Apenas uma noite de privação de sono é o suficiente para resultar em níveis aumentados de cortisol, aumentando, consequentemente, o estresse (Minkel, 2014). A restrição do sono de forma crônica pode aumentar a atividade do eixo HPA, gerando uma redução na área do córtex frontal e hipertrofia amigdalina, o que está diretamente relacionado a indivíduos mais impulsivos e ansiosos (Pires, et al., 2016).

De acordo com Coles et al. (2015), a interrupção do sono em jovens adultos está relacionada à ansiedade, pois estudos feitos demonstraram que pessoas que possuem problemas associados ao sono tiveram taxas mais altas de transtornos de ansiedade, Transtorno Obsessivo Compulsivo (TOC) e depressão. Esta interrupção também terá como consequência a alteração na expressão de emoções, como o aumento da instabilidade emocional e a irritabilidade. Além disso, a privação do sono aumenta a ansiedade em períodos de estresse, em comparação a indivíduos saudáveis, que possuem um sono adequado.

\subsection{Nutrientes no controle de sintomas}

\subsubsection{Vitaminas D e do complexo B}

A vitamina D (1,25-dihidroxivitamina) é conhecida por regular vários aspectos da morfologia do cérebro, fisiologia e comportamento, desempenhando um papel extremamente importante no sistema nervoso central. Várias linhas de evidência 
mostram que a vitamina D modula o desenvolvimento, neurotransmissão, neuroproteção e imunomodulação do cérebro (Cui, et al., 2017).

Estudos afirmam que baixos níveis plasmáticos podem prejudicar a síntese de dopamina e serotonina, essas pessoas que apresentam deficiência em vitamina D estão em maior risco de desenvolver depressão (Schaad, et al., 2019).

Um estudo realizado com 62 pacientes do sexo masculino e feminino com transtorno depressivo e transtorno de ansiedade, comparou os níveis de serotonina quando suplementados com vitamina D. Os indivíduos foram divididos em dois grupos: o primeiro grupo recebeu suplementação de colecalciferol vitamina D3 (50.000 UI) por 3 meses, tratamento farmacológico e suporte psicológico, e o segundo grupo recebeu este padrão de atendimento porém sem a suplementação de vitamina $\mathrm{D}$. O resultado da pesquisa demonstrou que ocorreu um aumento nos níveis de serotonina nos indivíduos suplementados com vitamina D, consequentemente, melhorando os sintomas dos transtornos mentais (Alghamdi, et al., 2019).

Outras vitaminas importantes são as do Complexo B que também são responsáveis metabolicamente na síntese de neurotransmissores do sistema nervoso central, sendo de extrema importância que seja consumido em quantidade adequada para um bom funcionamento do sistema nervoso central. Proteínas animais, leguminosas, hortaliças e frutas são alguns dos alimentos fonte de tais vitaminas. (Barbosa, et al., 2020).

\subsubsection{Magnésio}

Magnésio é um cofator com mais de 300 funções reconhecidas, algumas delas são: ligação hormonal ao receptor, contração muscular, atividade neural, liberação de neurotransmissores. O magnésio está relacionado com a atividade da serotonina e outros neurotransmissores, como também na função neuromuscular. Em estudos feitos com o processo do ansiolítico do magnésio consegue relaxar as artérias, abaixar a pressão arterial e manter os batimentos cardíacos de forma regular, ideal para pessoas estressadas e ansiosas (Sousa, et al., 2013).

Um estudo feito na Alemanha com camundongos concluiu que a deficiência de magnésio é responsável pelas mudanças no eixo hipotálamo-hipófise-adrenal auxiliando a hiper-emocionalidade, essas alterações fazem com que tenham comportamentos de ansiedade. Os efeitos usando o magnésio como forma de tratamento foram benéficos na melhora do quadro do comportamento de ansiedade nos camundongos (Sartori, et al, 2012).

\subsection{3 Ácidos graxos}

Os ácidos graxos são componentes essenciais das membranas celulares, que precisam ter uma fluidez adequada para acontecer a recepção de sinais elétricos. O ômega 3 tem como elementos o EPA (ácido eicosapentaenoico), que apresenta efeito anti-inflamatório, e, DHA (ácido docosahexaenoico), considerado o ácido graxo mais benéfico para a saúde do cérebro, responsável pela composição de neurônios cerebrais e processos cognitivos (Rocha \& Myva, 2020).

A meta-análise feita por Jama et al. no ano de 2018, observou que a suplementação de ômega 3 está associada à redução de sintomas ansiosos, em pacientes com o transtorno de ansiedade diagnosticado. Resultados positivos foram observados em dosagens maiores ou iguais que $2000 \mathrm{mg} /$ dia e com o consumo de EPA menor que $60 \%$.

Sementes, oleaginosas, óleos vegetais, peixes e folhas verdes escuras são alguns dos alimentos fontes de ômega 3 , cujo estudos apresentaram uma influência positiva nos quadros de ansiedade, pois possuem fitoquímicos alcalóides, flavonóides e glicosídeo com potencial tranquilizante amenizando os sintomas da doença. Já em pacientes depressivos apresentou melhora no foco e capacidade cognitiva, além da melhora do humor (Gonçalves, et al., 2017). 


\subsubsection{L-Triptofano}

A síntese da serotonina (5-HT) é feita através de um aminoácido essencial chamado de L-Triptofano que é responsável na ação de promover o bem-estar. Através dessa síntese gerada contribui em vários efeitos no Sistema Nervoso Central, como na regulação do humor, do estresse e da agressividade. Baixas taxas dessa síntese influenciam no aparecimento da ansiedade (Junior, et al., 2021).

Uma pesquisa feita por 10 mulheres do Distrito Federal avaliou que o triptofano ajuda no controle da ansiedade, compulsão e diminuição alimentar. Uma alimentação desequilibrada atrapalha o fornecimento de nutrientes para a realização do 5-HT, o que pode elevar os níveis de estresse oxidativos e depressão (Zanello, 2012).

\section{Conclusão}

Com o aumento de pessoas com ansiedade e depressão é necessário estar atento em relação aos hábitos de vida como a alimentação e a prática de exercícios físicos diários. Tendo em vista que, devido a ansiedade, as pessoas tendem a recorrer aos alimentos de forma compensatória, tentando amenizar todo aquele sintoma que foi provocado. Considerando que uma alimentação adequada e rica em vitaminas e minerais, noites de sono tranquilas e baixa ingestão de álcool faz com que o indivíduo tenha um controle do bem-estar mental e contribuindo para a saciedade.

Portanto, o acompanhamento nutricional feito por um nutricionista é de extrema importância, sabendo que o único profissional com propriedade em auxiliar o indivíduo em fazer uma prescrição dietética de todos os nutrientes necessários para o controle da ansiedade e depressão.

Outro fator importante nas pesquisas feitas recentemente em relação ao eixo intestino cerebral, é como o equilíbrio intestinal pode influenciar na função cerebral como também na parte imunológica, e como tudo isso pode estar relacionado a ansiedade e a depressão.

Diante do exposto nesta revisão, é necessário um maior aprofundamento em estudos sobre ansiedade, depressão e a neuronutrição, principalmente no atual momento em que se encontra a população mundial.

\section{Referências}

Alghamdi, S., Alsulami, N., Khoja, S., Alsufiani, H., Tayeb, H. O., \& Tarazi, F. I. (2020). Vitamin D Supplementation Ameliorates Severity of Major Depressive Disorder. Journal of molecular neuroscience: MN, 70(2), 230-235. 10.1007/s12031-019-01461-2.

Almada, L. F., Borges, M. F., \& Machado, S. E. C. (2014). Considerações neurobiológicas sobre a depressão maior - um histórico neurocientífico. Revista de Psicologia, 17 (26), 111-124. https://seer.pgsskroton.com/renc/article/view/2420.

Andrade, E. et al. (2018). L-Triptofano, ômega 3, magnésio e vitaminas do complexo B na diminuição dos sintomas de ansiedade. https://idonline.emnuvens.com.br/id/article/view/1165.

Aro, F. de, Pereira, B. V., \& Bernardo, D. N. D’ A. Comportamento alimentar em tempos de pandemia por Covid-19 Comportamento alimentar em tempos de pandemia por Covid-19 (2021). Brazilian Journal of Development. Curitiba, 7 (6), 59736-59748. 10.34117/bjdv7n6-386.

Bear, T., Dalziel, J. E., Coad, J., Roy, N. C., Butts, C. A., \& Gopal, P. K. (2020). The Role of the Gut Microbiota in Dietary Interventions for Depression and Anxiety. Advances in nutrition (Bethesda, Md.), 11(4), 890-907. 10.1093/advances/nmaa016.

Calhoon, G., \& Tye, K. Resolving the neural circuits of anxiety. Nat Neurosci 18, 1394-1404 (2015). 10.1038/nn.4101.

Cardoso, T. A., \& Chagas, L. D. M. (2019). Saúde do sono: a importância do sono no dia a dia. Caderno De Graduação - Ciências Exatas E Tecnológicas UNIT - SERGIPE, 5(2), 83. https://periodicos.set.edu.br/cadernoexatas/article/view/6685.

Coles, M. E., Schubert, J. R., \& Nota, J. A. (2015). Sleep, Circadian Rhythms, and Anxious Traits. Current psychiatry reports, 17(9), 73. 10.1007/s11920-0150613-X.

Cui, X., Gooch, H., Petty, A., McGrath, J. J., \& Eyles, D. (2017). Vitamin D and the brain: Genomic and non-genomic actions. Molecular and cellular endocrinology, 453, 131-143. 10.1016/j.mce.2017.05.035. 
D'ávila, L. I., et al. (2020). Processo patológico do transtorno de ansiedade segundo a literatura digital disponível em português - revisão integrativa. Revista Psicologia e Saúde, 12(2), 155-168. 10.20435/pssa.v0i0.922

Diniz, J., Neves, S. \& Vieira, M. (2020). Ação dos Neurotransmissores Envolvidos na Depressão. Ensaios e Ciência C Biológicas Agrárias e da Saúde. 24. 437443. 10.17921/1415-6938.2020v24n4p437-443.

Ekstrand, B., Scheers, N., Rasmussen, M. K., Young, J. F., Ross, A. B., \& Landberg, R. (2021). Brain foods - the role of diet in brain performance and health. Nutrition reviews, 79(6), 693-708. 10.1093/nutrit/nuaa091.

Fernandes, Helder Matheus, Souza, Ingrid \& Fernandes, Daniele. (2020). Neuronutrição na ótica da ansiedade e depressão: perspectivas durante o processo de envelhecimento. 1. 1-13. https://editorarealize.com.br/artigo/visualizar/73228.

Foster, J. A., \& McVey Neufeld, K. A. (2013). Gut-brain axis: how the microbiome influences anxiety and depression. Trends in neurosciences, 36(5), 305-312. 10.1016/j.tins.2013.01.005.

Garcia, L. P. \& Sanchez, Z. M (2020). Consumo de álcool durante a pandemia da COVID-19: uma reflexão necessária para o enfrentamento da situação. Cadernos de Saúde Pública. 36 (10). 10.1590/0102-311X00124520.

Gonçalves, P. F., Sousa, B. R. V. de, Maracajá, B. R. V. de, \& Veiga, D. K. E (2016). Alimentação funcional como suporte para melhor prognóstico da depressão. Anuais I CONBRACIS Campina Grande: Realize Editora, 0, 1-6. https://www.editorarealize.com.br/index.php/artigo/visualizar/18859

Hartley, C. A., \& Phelps, E. A. (2012). Anxiety and decision-making. Biological psychiatry, 72(2), 113-118. 10.1016/j.biopsych.2011.12.027.

Junior, Dourival Tavares Sousa, Verde, Thiago Ferreira Cândido Lima \& Landim, Liejy Agnes Santos Raposo (2021). Alimentos ricos em triptofano e seu efeito na liberação da serotonina e possíveis benefícios no transtorno de ansiedade. Research, Society and Development, 10 (14), 1-7. 10.33448/rsd-v10i14.22190.

Martin, E. I., Ressler, K. J., Binder, E., \& Nemeroff, C. B. (2009). The neurobiology of anxiety disorders: brain imaging, genetics, and psychoneuroendocrinology. The Psychiatric clinics of North America, 32(3), 549-575. 10.1016/j.psc.2009.05.004.

Meroni, M., Longo, M., \& Dongiovanni, P. (2019). Alcohol or Gut Microbiota: Who Is the Guilty? International journal of molecular sciences, $20(18), 4568$. 10.3390/ijms20184568.

Minkel, J., Moreta, M., Muto, J., Htaik, O., Jones, C., Basner, M. \& Dinges, D. (2014). Sleep deprivation potentiates HPA axis stress reactivity in healthy adults. Health psychology: official journal of the Division of Health Psychology, American Psychological Association, 33(11), 1430-1434. 10.1037/a0034219.

Organização mundial da saúde (2017). Depressão e outros transtornos mentais comuns: estimativas de saúde global. Global Health Estimates. https://apps.who.int/iris/bitstream/handle/10665/254610/W?sequence=1.

Organização mundial da saúde (2018). Global status report on alcohol and health. World Health Organization. https://www.who.int/substance_abuse/publications/global_alcohol_report/en/.

Organização pan-americana da saúde (2020). Uso de álcool durante a pandemia de COVID-19 na América Latina e no Caribe. Organização Pan-Americana da Saúde. Recuperado set 20, 2021, de https://iris.paho.org/handle/10665.2/52936.

Pires, G. N., Bezerra, A. G., Tufik, S., \& Andersen, M. L. (2016). Effects of acute sleep deprivation on state anxiety levels: a systematic review and metaanalysis. Sleep medicine, 24, 109-118. 10.1016/j.sleep.2016.07.019.

Rocha, A., Myva, L. \& Almeida, S. (2020). O papel da alimentação no tratamento do transtorno de ansiedade e depressão. Research, Society and Development. 9. 10.33448/rsd-v9i9.7890.

Roth, T., et al. (2020). Prejuízo na absorção de nutrientes pela ingestão de álcool: uma revisão. Research, Society and Development. 9. 190911910. 10.33448/rsdv9i1.1910.

Rufino, S., Leite, R. S., \& Freschi, L. (2018). Aspectos gerais, sintomas e diagnóstico da depressão. Revista Saúde em Foco, 10, 837-843. https://portal.unisepe.com.br/unifia/wp-content/uploads/sites/10001/2018/11/095_ASPECTOS-GERAIS-SINTOMAS-E-DIAGN\%C3\%93STICO-DADEPRESS\%C3\%83O.pdf.

Saneei, P., Hajishafiee, M., Keshteli, A., Afshar, H., Esmaillzadeh, A., \& Adibi, P. (2016). Adherence to Alternative Healthy Eating Index in relation to depression and anxiety in Iranian adults. British Journal of Nutrition, 116(2), 335-342. 10.1017/S0007114516001926.

Sartori, S. B., Whittle, N., Hetzenauer, A., \& Singewald, N. (2012). Magnesium deficiency induces anxiety and HPA axis dysregulation: modulation by therapeutic drug treatment. Neuropharmacology, 62(1), 304-312. 10.1016/j.neuropharm.2011.07.027.

Schaad, K. A., Bukhari, A. S., Brooks, D. I., Kocher, J. D., \& Barringer, N. D. (2019). The relationship between vitamin D status and depression in a tactical athlete population. Journal of the International Society of Sports Nutrition, 16(1), 40. 10.1186/s12970-019-0308-5.

Smith, D. G., et al. (2020). Association of food groups with depression and anxiety disorders. European journal of nutrition, 59(2), 767-778. 10.1007/s00394019-01943-4

Su, K. P., Tseng, P. T., Lin, P. Y., Okubo, R., Chen, T. Y., Chen, Y. W., \& Matsuoka, Y. J. (2018). Association of Use of Omega-3 Polyunsaturated Fatty Acids With Changes in Severity of Anxiety Symptoms: A Systematic Review and Meta-analysis. JAMA network open, 1(5), e182327. 10.1001/jamanetworkopen.2018.2327.

Tafet, G. E., \& Nemeroff, C. B. (2020). Pharmacological Treatment of Anxiety Disorders: The Role of the HPA Axis. Frontiers in psychiatry, 11, 443. $10.3389 /$ fpsyt.2020.00443. 
Research, Society and Development, v. 10, n. 17, e52101724454, 2021

(CC BY 4.0) | ISSN 2525-3409 | DOI: http://dx.doi.org/10.33448/rsd-v10i17.24454

Yogi, C. M., Lomeu, F. L. R. de O. \& Silva, R. R. (2018). Alimentação, Depressão e Ansiedade: entenda a relação. Universidade Federal de alfenas, Minas Gerais. https://docplayer.com.br/104955159-Alimentacao-depressao-e-ansiedade-entenda-a-relacao.html.

Zanello, D. R. de P. (2012). Efeitos do L- Triptofano sobre ansiedade, compulsão e escolha alimentar. Curso de Nutrição, Centro Tecnológico, Centro Universitário de Brasília, Brasília., 1, 1-23. https://repositorio.uniceub.br/jspui/bitstream/235/7287/1/Diogo\%20Rabelo\%20de\%20Paula\%20Zanello.pdf. 